\title{
Bkm sequences from the human $X$ chromosome contain large clusters of GATA/GACA repeats
}

\author{
R. P. ERICKSON, ${ }^{*}+$ C. E. ROSS, ${ }^{*}$ J. L. GORSKI, ${ }^{*} \dagger$ J. R. D. STALVEY* \\ AND M. M. DRUMM* \\ Departments of *Human Genetics and + Pediatrics and Communicable Diseases, \\ University of Michigan School of Medicine, Ann Arbor, Michigan 48109-0618
}

\section{SUMMARY}

In order to determine whether the regional localizations of $\mathrm{Bkm}$ repeats detected on the human X chromosome consisted of typical GATA/GACA repeats, clones were isolated, mapped, and sequenced. Nine Bkm-hybridizing clones from Kunkel's fluorescent-activated, cell-sorted $\mathrm{X}$-chromosome library were all unique. Five were mapped in detail with restriction enzymes and the Bkm-hybridizing segments were localized. Confirmation of $\mathbf{X}$ chromosomal homology was obtained for 2 of the clones and Bkm segments from these 2 clones were sequenced. Seventeen contiguous GATA repeats were found in each clone and the overall repeat arrangement showed relatively few differences from previously sequenced Bkm sequences. These are the first sequences of human $B \mathrm{~km}$ repeats. The results, when compared with previously published results, suggest that there may be significant differences between the organization of $\mathrm{Bkm}$ repeats on the human $\mathrm{X}$ and on the human $\mathrm{Y}$ chromosome.

\section{INTRODUCTION}

A satellite DNA sequence that appeared to be exclusive to the female, heterogametic sex was previously isolated from the venomous snake, the banded krait (Bungarus fasciatus) (Singh et al. $1980 a)$. When the banded krait minor satellite DNA $(\mathrm{Bkm})$ was hybridized to DNA of a range of snake species representing a wide spectrum of sex-chromosome evolution, it hybridized strongly to the $\mathrm{W}$ chromosome, the heterogametic chromosome. Bkm DNA was also found in a variety of other organisms. In Drosophila, $\mathrm{Bkm}$ is localized near the base of the $\mathrm{X}$ chromosome (Singh, et al. 1980 b), while in the mouse, Bkm sequences were found to be located near the centromere of the $Y$ chromosome (Jones \& Singh, 1981), and in studies with XX,Sxr mice, this region was shown to be strongly male determining (Singh \& Jones, 1982).

Restriction enzymes have shown little sex difference in Bkm hybridization patterns in humans (Jones \& Singh, 1981). In situ studies have shown that Bkm is concentrated on two regions of the $\mathrm{X}$ chromosome and on two autosomal chromosomes but relatively little was found on the $Y$ chromosome (Kiel-Metzger et al. 1985). This result was unexpected due to the presence of $\mathrm{Bkm}$ in the heterogametic chromosome of other mammals, such as mice. We were unable to find Bkm-positive human Y chromosomal cosmids when using the Drosophila-derived pCS316 Bkm-containing clone, either using salmon sperm DNA (Wolfe et al. 1984) or E. coli DNA (Erickson et al. 1987) as the non-specific competitor. In contrast, Arnemann et al. (1986) detected Bkm-positive human $\mathrm{Y}$ cosmids using a synthetic oligodeoxynucleotide probe. However, these cosmids seem to have only relatively short runs of GATA repeats, as 
determined by a non-sequencing technique, compared with mouse control clones. We have isolated Bkm-hybridizing clones from a fluorescent-activated, cell-sorted human X-chromosome library, characterized them by restriction mapping and sequencing, and shown that they contain long repeats and higher order structures as have been found in other mammalian Bkm-containing clones.

\section{MATERIALS AND METHODS}

A library of the human $\mathrm{X}$-chromosome, prepared by fluorescent-activated sorting, in the lambda bacteriophage Charon 21A vector was obtained from Dr Louis Kunkel (Kunkel et al. 1982). $E$. coli K802 was infected by the bacteriophage and about 10000 plaques were screened for the presence of Bkm with the Bkm-containing plasmid pCS316 obtained from a Drosophila library (provided by Dr Kenneth Jones) by standard techniques. Thirty-three plaques showed strong hybridization to the Bkm probe. Twenty were chosen at random of which nine were analysed. These nine were unique, as shown by comparing their restriction patterns with a variety of restriction enzymes (data not shown). Five were characterized in more detail.

The human inserts were separated from the bacteriophage DNA by digestion with HindIII and subsequent electrophoresis (about $2.5 \mu \mathrm{g}$ DNA per well) through a $0.9 \%$ agarose gel with recovery of the insert DNA on Schleicher \& Schuell NA45 paper per manufacturer's suggested protocols. Restriction enzymes were obtained commercially from Boehringer Mannheim, Bethesda Research Laboratories, International Biotechnologies, and P.L. Biochemicals and used per manufacturer's protocols. Location of restriction sites was performed by analysis of appropriate double digestions. Identification of Bkm-containing fragments was performed after Southern transfer (Southern, 1975). Prehybridization was in $50 \%$ formamide, $1 \%$ SDS, and $1 \mathrm{M}-\mathrm{NaCl}$ at $42^{\circ} \mathrm{C}$. Nick-translated pCS316 $\left(10^{7} \mathrm{cpm} / \mu \mathrm{g}\right)$ and sheared salmon sperm DNA (final concentration $50 \mu \mathrm{g} / \mathrm{ml}$ ) were denatured by boiling and added to the prehybridization mixture. After overnight hybridization, the filters were washed to $0.5 \times \mathrm{SSC}$ in $0.1 \% \mathrm{SDS}$ at $65^{\circ} \mathrm{C}$ and exposed to Kodak X-Omat AR at $-70^{\circ} \mathrm{C}$ with intensifying screens.

Mouse cell lines 1R (Nabholz et al. 1969) and HORL9X (1R with the addition of an intact human X chromosome (Goodfellow, 1975)) were the generous gift of Dr Peter N. Goodfellow. DNA was restricted to completion with EcoRI, transferred by the Southern technique and hybridized (as described above or in $1 \% \mathrm{SDS}, 1 \mathrm{M}-\mathrm{NaCl}$ at $65^{\circ} \mathrm{C}$ ) with nick-translated intact lambda or random oligodeoxynucleotide-primed probes (Feinberg \& Vogelstein, 1984) to the isolated lambda HindIII inserts. Those lambda clones which showed repetitive patterns with both cell lines' DNA were prehybridized to $\mathrm{C}_{0} \mathrm{~T} 100$ following the procedure of Litt \& White (1985). Briefly, freshly precipitated probe was added to $100 \mu \mathrm{g} / \mathrm{ml}$ of sonicated $(300-500 \mathrm{bp})$ human DNA in $5 \times \mathrm{SSC}$, boiled for $10 \mathrm{~min}$, cooled at $0{ }^{\circ} \mathrm{C}$ for $1 \mathrm{~min}$, and incubated at $68^{\circ} \mathrm{C}$ for $10 \mathrm{~min}$ before its addition to the filter and prehybridization mixture.

The $0.6 \mathrm{~kb}$ PstI-HindIII fragment of $\lambda \mathrm{X} 13$ and the $1.1 \mathrm{~kb}$ BamHI-HindIII fragment of $\lambda X 81$ were cloned into $\mathrm{M} 13$ vectors $\mathrm{mp} 18$ and $\mathrm{mp} 19$ in both orientations and sequenced by the method of Sanger et al. (1977), using T7 polymerase from U.S. Biochemical as recommended (Taber \& Richardson, 1987), and using sequentially loaded wedged polyacrylamide gels. Dideoxynucleotide triphosphates and deoxynucleotide triphosphates were purchased from P.L. 


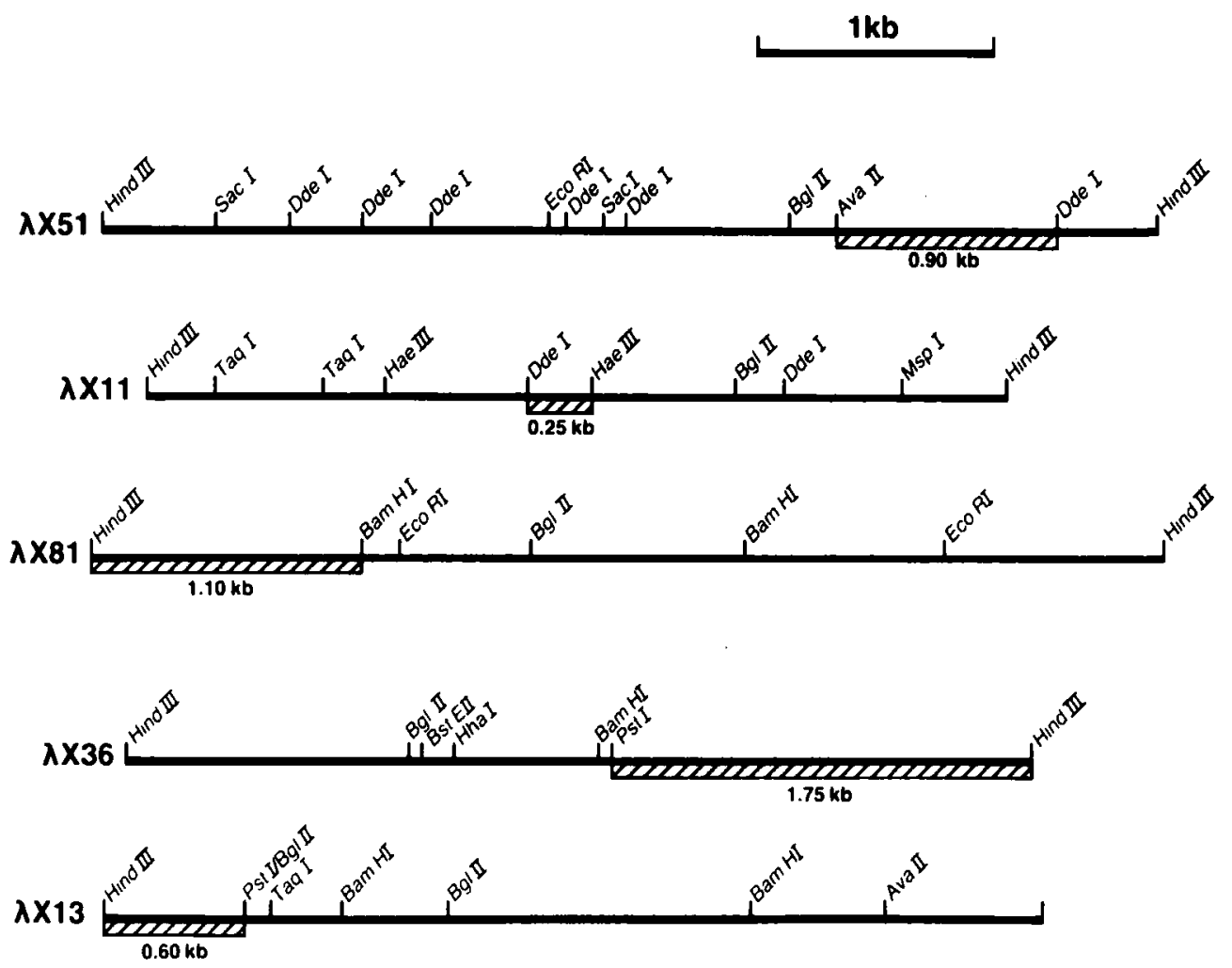

Fig. 1. Restriction maps and Bkm-hybridizing fragments of $\lambda$ clones isolated with the Drosophila Bkm plasmid, pCS316.

Biochemicals. $\alpha^{-32} \mathrm{P}$-dATP of $400 \mathrm{Ci} / \mu$ mole was purchased from Amersham. The 615 bp PstIHindIII fragment of $\lambda \mathrm{X} 13$ was completely sequenced; $30 \%$ was sequenced off both strands. $206 \mathrm{bp}$, starting $73 \mathrm{bp}$ from the $5^{\prime}$ HindIII site and including the GATA repeats, were sequenced from the BamHI-HindIII fragment of $\lambda$ X81.

\section{RESULTS}

Of the 10000 plaques screened for $\mathrm{Bkm}, 33$ were chosen that showed strong hybridization. Nine of these $-\lambda \mathrm{X} 11, \lambda \mathrm{X} 13, \lambda \mathrm{X35}, \lambda \mathrm{X36}, \lambda \mathrm{X} 51, \lambda \mathrm{X} 54, \lambda \mathrm{X} 57, \lambda \mathrm{X81}, \lambda \mathrm{X} 101$ - have been plaque purified and shown to be unique by comparing their restriction patterns with specific enzymes (data not shown). Five bacteriophage have been mapped by restriction analysis and Bkmhybridizing fragments identified. The results of the $\lambda$ X11, $\lambda$ X13, $\lambda$ X36, $\lambda$ X51 and $\lambda$ X81 bacteriophage insert characterizations are illustrated in Fig. 1. The $\lambda$ X11 insert is $3 \cdot 6 \mathrm{~kb}$ in length with its $250 \mathrm{bp} \mathrm{Bkm-hybridizing} \mathrm{fragment} \mathrm{located} \mathrm{near} \mathrm{the} \mathrm{centre} \mathrm{of} \mathrm{the} \mathrm{insert} \mathrm{and}$ bordered by DdeI and HaeIII restriction sites. The $\lambda \mathrm{X} 13$ insert is $4.0 \mathrm{~kb}$ in length with a $600 \mathrm{bp} \mathrm{Bkm-hybridizing} \mathrm{fragment} \mathrm{bounded} \mathrm{by} \mathrm{a} \mathrm{PstI} \mathrm{site} \mathrm{and} \mathrm{one} \mathrm{of} \mathrm{the} \mathrm{HindIII} \mathrm{cloning} \mathrm{sites.}$ The $3.8 \mathrm{~kb} \lambda \mathrm{X} 36$ insert was restriction mapped and found to have a $1.75 \mathrm{~kb}$ Bkm-hybridizing region which is located between a HindIII cloning site and a PstI restriction site. The $\lambda$ X51 insert is $4.4 \mathrm{~kb}$ long and contains a Bkm-hybridizing fragment of $900 \mathrm{bp}$ which is bounded by 


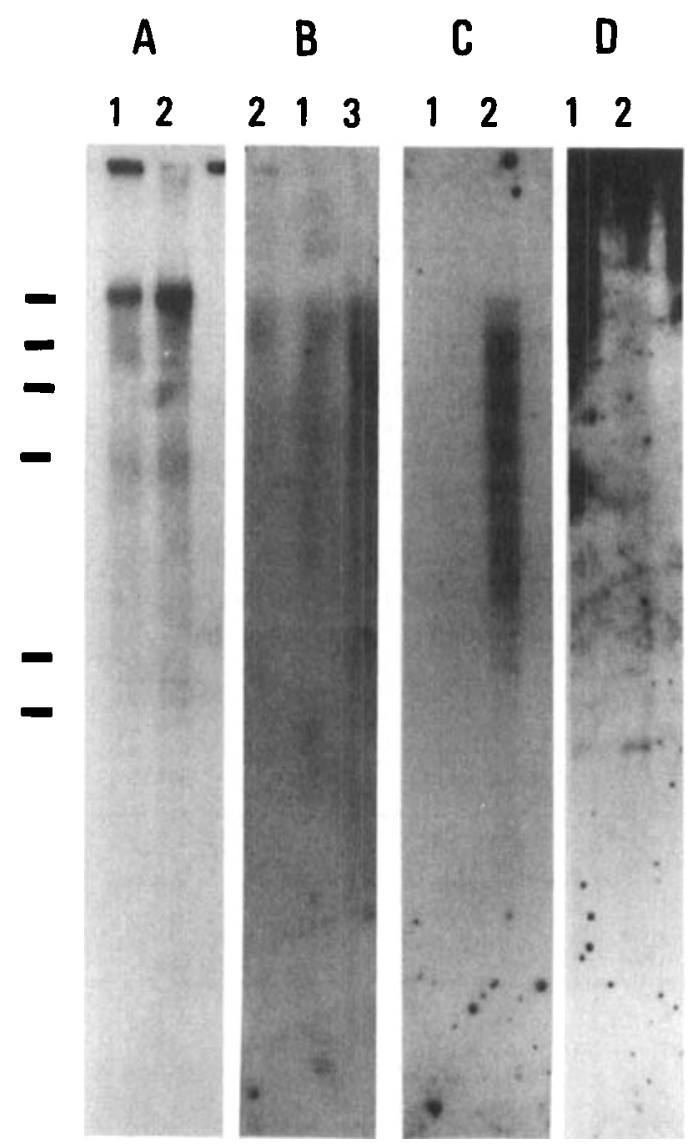

Fig. 2. Hybridization of $\lambda \mathrm{X}$ Bkm clones to mouse DNA or mouse thuman X DNA. DNA was restricted with $E c o R I$, electrophoresed, transferred to Genescreen Plus ${ }^{\text {TM }}$ by the Southern technique, and hybridized with or without preassociation to sonicated human DNA, (A) $\lambda$ X51, $0.5 \times$ SSC, without preassociation, (B) $\lambda \mathrm{X51}, 0.5 \times \mathrm{SSC}$, with preassociation, (C) $\lambda \mathrm{X13}, 0.2 \times \mathrm{SSC}$, without preassociation and (D) $\lambda$ X81, $0.2 \times \mathrm{SSC}$, without preassociation. 1, 1R; 2 , HORL9X; 3, control human female DNA.

AvaII and DdeI restriction sites. The $\lambda \mathrm{X} 81$ insert is $4.5 \mathrm{~kb}$ long with its $1.10 \mathrm{~kb} \mathrm{Bkm}$ hybridizing fragment located between the HindIII cloning site and a BamHI restriction site.

\section{Chromosomal assignment of clones}

Even though the flow-sorted library has shown a preponderance of X-derived clones (Kunkel et al. 1982), we examined the chromosomal assignments of the clones we characterized. The clones showed a variety of patterns of hybridization to the DNAs of a mouse cell line $(1 \mathrm{R})$ and a mouse-human somatic cell hybrid with only the human X chromosome (HORL9X)(Fig. 2). Inasmuch as $\mathrm{Bkm}$ sequences are also abundant in mice, some clones gave similar patterns with these two DNAs. $\lambda$ X51 hybridized to a large band and also gave a smear and a faint repeat ladder with the mouse DNA whether or not the human $X$ was present (Fig. 2A). Prehybridization to sonicated human DNA eliminated the large band in both cell lines, leaving faint smears with the mouse DNA and mouse with human X DNA and a stronger smear with control human DNA (Fig. 2 B). These results suggest that the large MW band detected by $\lambda$ X51 
Sequence of humanXBkm13

1

AGCTTTGGTT CAATTTTTAT GGTTTTTATG GAAGgTACAA AgTTACTAAT GCACCACCCC

61 acagtaAgca cCagccccac atggtTtcat agagaAatat tagaAaAtct tcaAagattg

121 GGTAGTTGTG ATGCTACGAA AACTTTTCCA AAACAAACTC CTACTGAAGT TTTATGAAAT

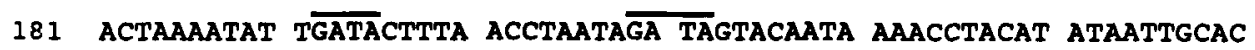

241 TTATGgatat AGATGTACTA ATTAAAATAC TAGCAAACAT AaCcCAACAC TGCATTCA $\overline{G A}$

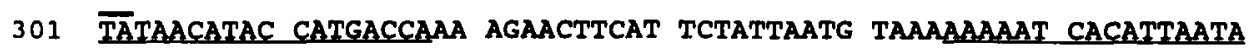

361 GAATTAAGGG GAAACATAT GATTATCTAC AGTGATGCTA AATGAAACCA ACAATATTTA

421 AAATTATTCT GATAAGACCC TCAAGAACTT GAATTGATGG AGATTCAACT ATATATATAT

481 AAATATATGT TTATAAATAT AGATAGACAG ATAGATAGAT AGACAGATAG ATAGATAGAT

541 AGATAGATAG ATAGATAGAT AGATAGATAT AGATATAGAT ACTTTAATCC TAAAGGCAAG

601 ATCTTACCTA CTGCA

Sequence of humanXBkm81

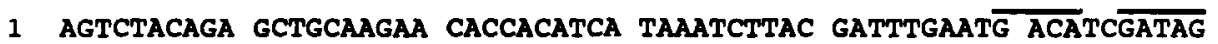

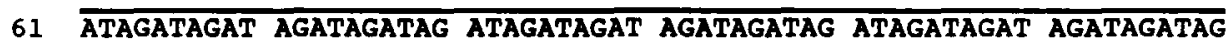

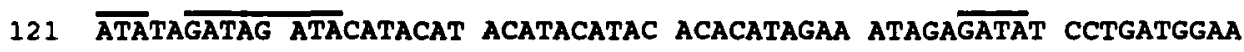

181 agataAacc atgagtctgg taAtag

Fig. 3. Sequences of $\lambda \times 13$ and $\lambda$ X81 fragments with GATA repeats. Sequences read from both strands are underlined, GATA/GACA repeats are overlined.

is a repeated element other than $\mathrm{Bkm}$, while the diffuse smear could be due to $\mathrm{Bkm}$. At high stringency, $\lambda \mathrm{X} 13$ hybridized as a smear, with faint bands suggestive of unique sequences, only with the somatic cell hybrid (containing the human X) DNA (Fig. 2C). Thus, $\lambda$ X13 contains repeat sequences that hybridize to the human $X$, but not to mouse DNA, at this stringency. $\lambda$ X81 demonstrated unique sequence bands with the somatic cell hybrid DNA (containing the human DNA) after high stringency washing $(0 \cdot 2 \times \mathrm{SSC})$ but not with the parental mouse line (Fig. 2D). Thus, $\lambda \mathrm{X} 13$ and $\lambda \mathrm{X81}$ contain human $\mathrm{X}$ homologous sequences, as predicted for members of this library, although they still could be derived from other human chromosomes. A definite determination could not be made for $\lambda$ X51 (Fig. $2 \mathrm{~A}, \mathrm{~B}$ ) and $\lambda \mathrm{X} 11$ and $\lambda \mathrm{X36}$ (data not shown).

The 615 bp $\lambda$ X13 Bkm-hybridizing fragment contained 23 GATA/GACA repeats in a span 
of $390 \mathrm{bp}$ (Fig. 3). Repeats 21, 22, and 23 were separated by the dinucleotide, TA, and abundant TATA repeats preceded the major block of GATA/GACA repeats. The $68 \mathrm{bp}$ of contiguous repeats were preceded by ATA. Reading $5^{\prime}$ to $3^{\prime}$, an open reading frame of $150 \mathrm{bp}$ at $28-177$ precedes the GATA/GACA repeats but does not have consensus codons flanking the initiation site to suggest likely usage. There are other shorter open reading frames (131-193, 243-305, 457-522). An in-frame start codon for the $3^{\prime}-5^{\prime}$ complementary strand which would lead into the tyr leu ser leu hydrophobic repeat potentially coded by the complement to the GATA repeats was not seen, but the fragment only extended $29 \mathrm{bp}$ further in this direction. The 206 bp of the $\lambda$ X81 Bkm-hybridizing fragment contained 21 GATA/GACA repeats, again with a separation by the dinucleotide, TA. A start codon in the complementary strand immediately precedes the 20th repeat complement and remains in frame through the 20 repeats to bp 37 . Thus, although the Drosophila $\mathrm{Bkm}$ clone used to find these clones might have contained other repeats (two Drosophila clones closely related to the one we used have been sequenced and did not contain other repeats; Singh et al. 1984), the sequenced human clones did not have detectable repeats other than the GATA/GACA, Bkm-related repeats.

\section{DISCUSSION}

The hypothesis that $\mathrm{Bkm}$ is involved in sex differentiation originated in work by Singh et al. (1976). Analytical equilibrium centrifugation was done on the venomous banded krait which has heteromorphic sex chromosomes. A minor satellite unique to the heterogametic, female sex was discovered (Singh et al. 1976). Radioactive banded Krait minor satellite DNA (Bkm) was then hybridized to DNA of ten species of snakes. These snakes represented all stages of sexchromosome evolution. With the exception of the evolutionarily primitive boa constrictor, significantly more Bkm hybridized to female DNA than to male DNA. In situ studies showed that the Bkm homologous regions were located throughout the genome, but they were especially abundant on part or all of the $W$ chromosome.

Mammals broke away from the evolutionary line long before the lineages of birds and reptiles appeared. Therefore, the presence of Bkm in the heterogametic sex in mammals could support the hypothesis that $\mathrm{Bkm}$ is universally involved in sex determination. In mice, Singh et al. $(1980 b)$ found by in situ hybridization that Bkm was localized proximal to the centromere on the telomeric $Y$. Also, a sex specific pattern emerged when genomic DNA was digested with HaeIII, electrophoresed, blot transferred, and hybridized with $\mathrm{Bkm}$ : male DNA had a large molecular weight $\mathrm{Bkm}$ fragment which contained many $A l u \mathrm{I}$ restriction sites that was absent in the female. When DNA from XX phenotypic males (XX,Sxr) was restricted, blot transferred, and hybridized with Bkm, the pattern was nearly identical to that of the normal male mouse. Using in situ studies, Singh \& Jones (1982) demonstrated that the XY,Sxr male carrier possesses an inherited aberrant $\mathrm{Y}$ chromosome with two Bkm homologous regions, one region proximal to the centromere as in a normal $Y$ chromosome plus an unexpected region at the tip of the $\mathrm{Y}$ chromosome. This second region was shown to be the result of translocation (Evans et al. 1982). Singh and Jones (1982) concluded that the XX,Sxr male is the result of a cross-over between the distal ends of the $\mathrm{X}$ and the $\mathrm{Y}$ chromosomes which transfers a male determining $\mathrm{Bkm}$ region to the $\mathrm{X}$ chromosome, an interpretation of $S x r$ which was also suggested by electron microscopical and immunological studies (Shapiro et al. 1982). 
This information on the morphologic location of $\mathrm{Bkm}$ in various organisms led to the assertion that Bkm was evolutionarily conserved. Many investigators then turned to sequencing $\mathrm{Bkm}$ and its flanking regions in the hope of confirming the hypothesis. Sequencing experiments found the highly repetitive Bkm to consist of multiple copies of GATA or GACA. Sequences of Bkm containing clones of Elaphe radiata (Epplen et al. 1982), Mus musculus (Epplen et al. 1983; Singh et al. 1984) and Drosophila melanogaster (Singh et al. 1980a, b) were determined individually and Levinson et al. (1985) pooled these data to analyse them. Long clusters of GATA and GACA are common to $M$. musculus and D. melanogaster but the similarities between the two organisms' clusters ends there. The number of tandem GATA or GACA repeats per cluster varies between the two organisms and the disruptions between these tandem repeats are not similar. Also, the mouse Bkm has GATA and GACA regions intermixed while Drosophila has distinct tandem arrays of GATA and GACA. This evidence suggests that Bkm sequences are not evolutionarily conserved regions, but rather are developed by convergent evolution in which there could be selection favouring Bkm's evolution (Levinson et al. 1985; Platt \& Dewey, 1987). However, in the Mediterranean meal moth, Ephestia kuehniella, Bkm sequences form autosomal hypervariable DNA loci which, it was suggested, may rapidly vary by transposition of mobile elements (Traut, 1987). Thus, rapid evolution of Bkm sequences without selection for function is also possible.

The hypothesis that $\mathrm{Bkm}$ sequences play a role in sex determination was a generalization from the occurrence of high concentrations of these GATA/GACA repeats on heterogametic chromosomes in snakes and mice. We found much lower relative concentrations of Bkm sequences in primate DNA than in mouse DNA (Erickson et al. 1987) and a relative paucity of in situ hybridization to the human Y (Kiel-Metzger et al. 1985). Using a different Drosophila Bkm clone for in situ hybridization, Singh \& Jones (1986) reported relatively more grains on the human $\mathrm{Y}$ chromosome than we had but no quantitation was given. We had screened over 100 human Y-chromosome-derived cosmids with the same clone used for our in situ studies without finding a positive cosmid (Wolfe et al. 1984; Erickson et al. 1987). Arnemann et al. (1986) used (GATA) $)_{4}$ and (GACA) $)_{4}$ synthetic oligodeoxynucleotides to identify a number of hybridizing human $Y$ cosmids. The maximum tandem repeats they found in the human $Y$ cosmids were much shorter $(n=12)$ than those characteristic of mice ( $n=32$ and $n=80$ in the controls used). We asked whether human X-chromosome Bkm clones would be more typical of other mammalian Bkm clones than those found on the human $\mathrm{Y}$.

Our studies have characterized Bkm-hybridizing DNA from a flow-sorted human Xchromosome library. Table 1 shows a summary of previously published characterizations of Bkm homologous DNA in E. radiata, D. melanogaster and M. musculus (Epplen et al. 1982, 1983; Singh et al. 1984) and our results. The lengths of Bkm homologous regions that we have isolated $(0 \cdot 25-1.75 \mathrm{~kb})$ are similar to the lengths of GATA/GACA regions in other organisms. Although the lengths of repeats are similar, the contents of contiguous regions appear to be somewhat different in humans than in other organisms. Epplen et al. $(1982,1983)$ obtained very fine restriction maps of Bkm clones from $E$. radiata and $M$. musculus (the largest fragments in the map were about $0 \cdot 2 \mathrm{~kb}$ ) and restriction sites were found intrinsic to the GATA/GACA repeat regions. An extreme example of this is in E. radiata which has a GATA/GACA repeat spanning 189 base pairs containing only two short non-repeat regions. Despite this, there still exists an AluI restriction site in the middle of the $80.4 \%$ GATA/GACA region. In our experiments, we 


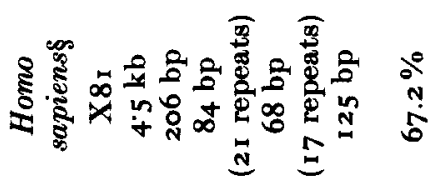

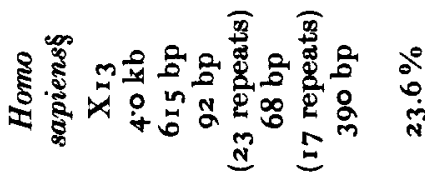

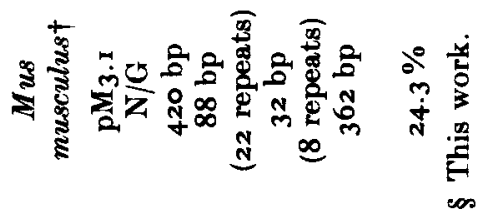
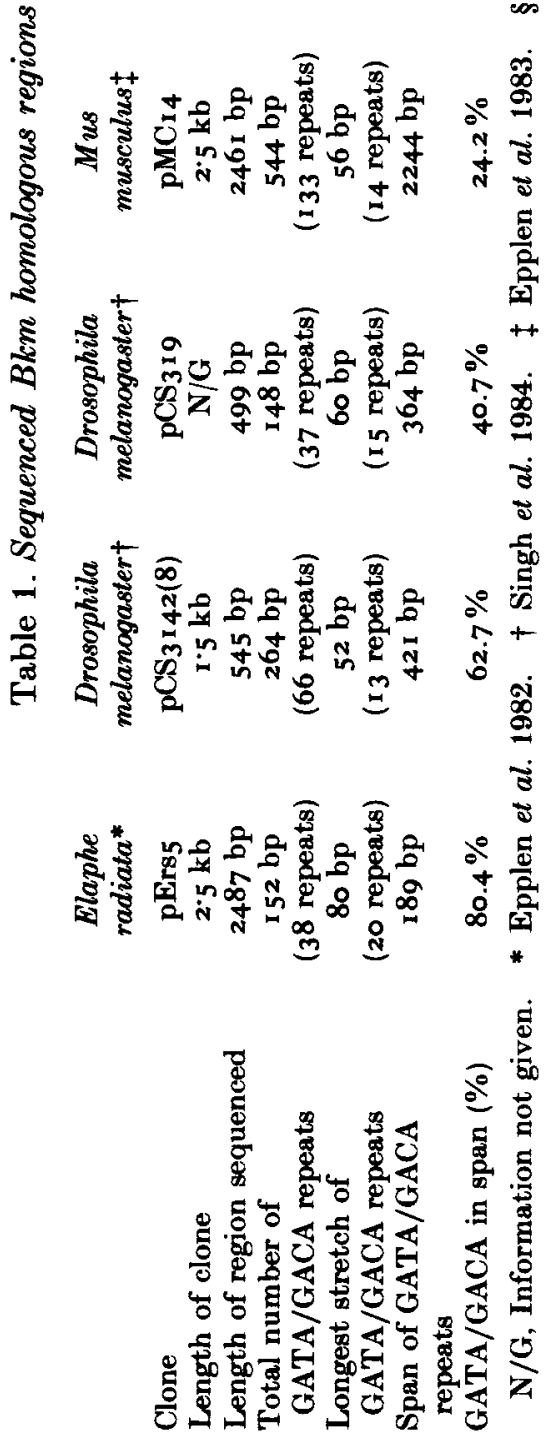
used a similar set of enzymes as Epplen et al. (1982, 1983) but obtained maps with fewer restriction sites (Fig. 1). Although the non-repetitive regions seem to be dissimilar, it appears that $\mathrm{Bkm}$ homologous regions in humans are about the same length as those in other organisms.

The sequence characterization of these clones revealed similarities to previously sequenced GATA/GACA repeats (Table 1). This is in apparent contrast to the results found by Arnemann et al. (1986) for Bkm-containing clones from the human $\mathrm{Y}$ chromosome which used an assay based on ligation of a synthetic oligodeoxynucleotide, (GATA) $)_{4}$, hybridized to the cosmids. Since the conditions of hybridization were not given, it is possible that single base pair mismatches could have been tolerated and that the apparent maximum of 12 repeats was not perfect. Only sequence characterization of GATA/GACA repeats from the $\mathrm{Y}$ chromosome can clarify their nature. This first sequence characterization of human Bkm sequences, from the $X$ chromosome, shows them to be similar to previously characterized mammalian, insect and snake Bkm sequences.

This work was supported by a NIH grant HD 20670. Dr J. L. Gorski is the recipient of NICHD Physician Scientist Scholar Award K11 HD 000788. We thank Dr L. Kunkel for the human X chromosome library and Dr P. N. Goodfellow for DNAs from cell lines. We also thank Dr Margaret Lomax and Ms Grace Jung for help in the initial sereening and Mrs Ann Mogan for preparing the manuscript.

\section{REFERENCES}

Arnemann, J., Jakubiczka, S., Schmidtke, J., Schäfer, R. \& Epplen, J. T. (1986). Clustered GatA repeats (Bkm sequences) on the human Y chromosome. Hum. Genet. 73, 301-303.

Epplen, J. T., Cellini, A., Romero, S. \& Ohno, S. (1983). An attempt to approach the molecular mechanisms of primary sex determination: $W$ - and Y-chromosomal conserved simple repetitive DNA sequences and their differential expression in mRNA. J. Exp. Zool. 228, 305-312.

Epplen, J. T., McCarrey, J. R., Sutou, S. \& Ohno, S. (1982). Base sequence of a cloned snake W-chromosome DNA fragment and identification of a male-specific putative mRNA in the mouse. Proc. Natn. Acad. Sci. USA 79, 3798-3802.

Erickson, R. P., Bevilacqua, A., Ross, C., Donaldson, S. \& Stalvey, J. R. D. (1987). Do Bkm sequences play a role in human sex determination? In Genetic Markers of Sex Differentiation (ed. F. P. Haseltine, M. E. MeClure and E. H. Goldberg), pp. 149-159. New York: Plenum Press.

Evans, E. P., Burtenshaw, M. D. \& Cattanach, B. M. (1982). Meiotic crossing over between the $X$ and $Y$ chromosomes of male mice carrying the sex-reversing $(S x r)$ factor. Nature $300,443-445$.

Feinberg, A. P. \& Vogelstein, B. (1984). Addendum: A technique for radiolabelling DNA restriction endonuclease fragments to high specific activity. Anal. Biochem.137, 266-267.

Goodfellow, P. N. (1975). Genetics and biochemistry of tissue antigens. D. Phil. Thesis, Oxford University.

Jones, K. W. \& Singh, L. (1981). Conserved repeated DNA sequences in vertebrate sex chromosomes. Hum. Genet. 58, 46-53.

Kiel-Metzger, K., Warren, G., Wilson, G. N. \& Erickson, R. P. (1985). Evidence that the human Y chromosome does not contain clustered DNA sequences $(\mathrm{Bkm})$ associated with heterogametic sex determination in other vertebrates. $N$. Engl. J. Med. 313, 242-245.

Kunkel, L. M., Tantraraki, U., Eisenhard, M. \& Latt, S. A. (1982). Regional localization on the human X of DNA segments cloned from flow sorted chromosomes. Nucleic Acids Res. 10, 1557-1578.

Levinson, G., Marsh, J. L., Epplen, J. T. \& Gutman, G. A. (1985). Cross-hybridizing snake satellite, Drosophila, and mouse DNA sequences may have arisen independently. Mol. Biol. Evol. 2, 494-504.

LiTT, M. \& WhiTe, R. L. (1985). A highly polymorphic locus in human DNA revealed by cosmid-derived probes. Proc. Natn. Acad. Sci. USA 82, 6206-6210.

Nabholz, M., Miggiano, V. \& Bodmer, W. F. (1969). Genetic analysis with human-mouse somatic cell hybrids. Nature 223, 358-363.

Platt, T. H. K. \& Dewey, M. J. (1987) Multiple forms of male specific simple repetitive sequences in the genus Mus. J. Mol. Evol. 25, 201-206.

Sanger, F., Nicklen, S. \& Coulson, A. R. (1977). DNA sequencing with chain-terminating inhibitors. Proc. Natn. Acad. Sci. USA 74, 5463-5467. 
Shapiro, M., Erickson, R. P.. Lewis, S. \& Tres, L. L. (1982). Serological and cytological evidence for increased Y-chromosome related material in $S x r$, XY (sex-reversed carrier, male) mice. J. Reprod. Immunol. 4, 191-206.

Singh, L. \& Jones, K.W. (1982). Sex reversal in the mouse Mus musculus is caused by a recurrent nonreciprocal crossover involving the $X$ and an aberrant $Y$ chromosome. Cell 28, 205-216.

Singh, L. \& Jones, K. W. (1986). Bkm sequences are polymorphic in humans and are clustered in pericentric regions of various acrocentric chromosomes including the Y. Hum. Genet. 73, 304-308.

Singh, L., Prmulips, C. \& Jonms, K. W. (1984). The conserved nucleotide sequences of Bkm, which define $S x r$ in the mouse, are transcribed. Cell 36, 111-120.

Singh, L., PUrdom, I. F. \& Jones, K. W. (1976). Satellite DNA and evolution of sex chromosomes. Chromosoma 59, 43-62.

Singh, L., Purdom, I. F. \& Jones, K. W. (1980a). Sex chromosome associated satellite DNA : evolution and conservation. Chromosoma 79, 137-157.

Singh, L., Purdom, I. F. \& Jon ms, K. W. (1980b). Conserved sex-chromosome-associated nucleotide sequences in eukaryotes. Quant. Biol. Cold Spring Harbor Symp. 45 (pt.2), 805-814.

SoUTHERN, E. (1975). Detection of specific sequences among DNA fragments separated by gel electrophoresis. J. Mol. Biol. 98, 503.

Taber, S. \& Richardson, C. C. (1987). DNA sequence analysis with a modified bacteriophage T7 DNA polymerase. Proc. Natn. Acad. Sci. USA 94, 4767-4771.

Traut, W. (1987). Hypervariable Bkm DNA loci in a moth, Ephestia kuehniella: Does transposition cause restriction fragment length polymorphism? Genetics 115, 493-498.

Wolfe, J., Erickson, R. P., Rigby, P. W. J. \& Goodflllow, P. N. (1984). Cosmid clones derived from both euchromatic and heterochromatic regions of the human $\mathrm{Y}$ chromosome. EMBO J. 3, 1997-2003. 